\title{
Characteristics of Solar Microwave Bursts Associated with CMEs: a Statistical Study
}

\author{
Chengwen Shao ${ }^{1,2,4} \dagger$,Min Wang ${ }^{1,2}$, Ruixiang Xie ${ }^{1,2}$, Qijun Fu ${ }^{2}$, \\ Yu-ying $\mathbf{L i u}^{2}$, Cheng-ming $\mathbf{T a n}^{2,4}$, Ying-na $\mathbf{S u}^{3,4}$ and Yuan $\mathbf{M a}^{1,2}$ \\ ${ }^{1}$ National Astronomical Observatory/Yunnan Observatory,CAS,Kunming,China.650011 \\ email: cwshaoanhui@etang.com,cwshaoanhui@163.com \\ ${ }^{2}$ National Astronomical Observatory,Chinese Academy of \\ Sciences(CAS),Beijing,China.100012. \\ ${ }^{3}$ Purple Mountain Observatory,Chinese Academy of Sciences(CAS),Nanjing,China. \\ ${ }^{4}$ Graduate School of Chinese Academy of Sciences(CAS),Beijing,China.100012.
}

\begin{abstract}
We selected 133 solar microwave bursts (SMBs) recorded by SGD and 133 CMEs observed by SOHO/LASCO from November 1999 through September 2003. These SMBs are associated with CMEs and flares. We analyzed the characteristics of the SMBs, including duration, flux peak, burst type, and spectral index. Correlated events were distinguished by time and the location of flare associated with the SMB. We find that (1)The duration of SMBs associated with narrow $\left(0^{\circ}<\right.$ width $\left.<20^{\circ}\right) /$ normal CMEs $\left(20^{\circ}<\right.$ width $\left.<120^{\circ}\right)$ or slow CMEs is below 40 minutes. (2)The duration of SMBs associated with Halo-like CMEs $\left(120^{\circ}<\right.$ width $\left.<360^{\circ}\right)$ or fast CMEs is from several minutes to 200 minutes. (3)The flux peak of SMBs associated with narrow CMEs/normal CMEs or slow CMEs is below 400sfu. (4)The flux peak of SMBs associated with Halo-like CMEs or fast CMEs is from several sfu to several thousands sfu. (5)the majority of SMBs, which are associated with Full Halo CMEs, are Complex/GB bursts. The majority of SMBs, which are associated with narrow CMEs/normal CMEs, are simple bursts. (6)U-shape spectra are observed. The spectra of SMB associated with CME is very flat when $f>f_{\max }$. A statistical result suggest that CME/flares and SMBs is probably a different manifestation of the same physical process. CME/flare and SMB have intrinsically a physical relationship.
\end{abstract}

Keywords. Sun: coronal mass ejections (CMEs), radio radiation

\section{Introduction}

The aim of this paper is to study the observational characteristics of SMBs associated with CMEs, including duration, peak flux, burst type and the spectral index. Section II is data selection. Section III is statistical results. Section IV is conclusions and discussions.

\section{Data Selection}

The CMEs of this study are available on the world wide web*. SMB data are available in SGD. Spectral index is studied by using NoRP XDR data.

Firstly, temporal association: We recorded the time $\mathrm{T}\left(\mathrm{R}_{s}\right)$ when the CME initiated from $1 \mathrm{R}_{s}$. The CME is associated with a flare if the flare starts in the time window $\mathrm{T}\left(\mathrm{R}_{s}\right) \pm 30 \mathrm{~min}$ (Zhou,G.P.et al. 2003). Secondly, spatial association: the CME is associated with a flare if the location of flare lies in the range of the CME span (M.Zhang 2002, Harrison et al. 1986).

$\dagger$ Present address: National Astronomical Observatory/Yunnan Observatory, Kunming, China. 

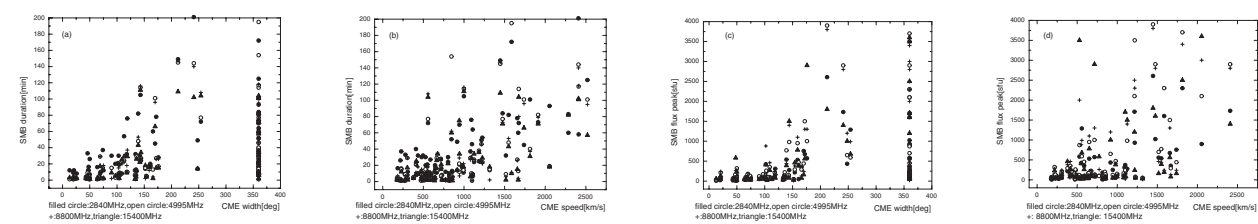

Figure 1. The relationship between SMB duration/flux peak and CME speed and width

The CME is associated with the flare if the CME and the flare satisfy the above two criteria simultaneously. The SMB, which is accompanied by a flare, is associated with the CME.

\section{3. statistical results and discussions}

According to the above two criteria, we selected $133 \mathrm{CMEs}$ and their associated SMBs. We analyzed the relationship between the duration/flux peak of SMBs and CME width/speed. The burst type of SMBs and spectral index were analyzed.

(1)The relationship between duration/peak flux of SMBs and CME width/speed. As seen from the figure 1, both long-duration and short-duration SMBs are accompanied by CMEs. Both great/intense bursts and small bursts are accompanied by CMEs.

(2)The burst type of SMBs associated with CMEs. The simple burst associated with narrow/normal CME in $2840 \mathrm{MHz}, 4995 \mathrm{MHz}, 8800 \mathrm{MHz}, 15400 \mathrm{MHz}$ is $72 \%, 88 \%, 86 \%$, $85 \%$ respectively. The complex burst associated with Full Halo CME in $2840 \mathrm{MHz}, 4995 \mathrm{MHz}$, $8800 \mathrm{MHz}, 15400 \mathrm{MHz}$ is $68 \%, 72 \%, 65 \%, 69 \%$ respectively.

(3)Spectral index are studied. The spectral index is between 0 and 1.5. This is consistent with Fu's study (Fu2004).

This paper's conclusion is basically consistent with that of Dougherty(2002). The characteristics of SMBs also are studied in this paper. This paper's conclusions are as follows: Both long-duration and short-duration SMBs are accompanied by CMEs. Both great/intense bursts and small bursts are accompanied by CMEs. The majority of these SMBs accompanied by Full Halo CME are Complex bursts. The majority of SMBs accompanied by narrow/normal CME is simple, uncomplicated bursts. A statistical result suggests that CME/flares and SMBs are the different manifestations of the same magnetic process.

\section{Acknowledgements}

The work is supported by the National NSF of China grant(NO.19833050, 19973016 and 10333030), the 973 project and the foundation of the CAS.

\section{References}

Dougherty et al. 2002,ApJ,577,457

Fu,Qi-jun 2004,solar radio astronomy symposium,Chengdu,China/personal communication

Harrison et al. 1986,A\&A,162,183

Zhang,M. et al. 2002,ApJ,574,L97-100

Zhou,G.P. et al. 2003,A\&A,397,1057

*http://cdaw.gsfc.nasa.gov/cme_list 